WellBeing International

WBI Studies Repository

8-2015

\title{
Variation in Brain Morphology of Intertidal Gobies: A Comparison of Methodologies Used to Quantitatively Assess Brain Volumes in
} Fish

Gemma E. White

Macquarie University

Culum Brown

Macquarie University

Follow this and additional works at: https://www.wellbeingintlstudiesrepository.org/acwp_vsm

Part of the Animal Structures Commons, Animal Studies Commons, and the Veterinary Anatomy Commons

\section{Recommended Citation}

White, G. E., \& Brown, C. (2015). Variation in brain morphology of intertidal gobies: a comparison of methodologies used to quantitatively assess brain volumes in fish. Brain, behavior and evolution, 85(4), 246-257.

This material is brought to you for free and open access by WellBeing International. It has been accepted for inclusion by an authorized administrator of the WBI Studies Repository. For more information, please contact wbisr-info@wellbeingintl.org.

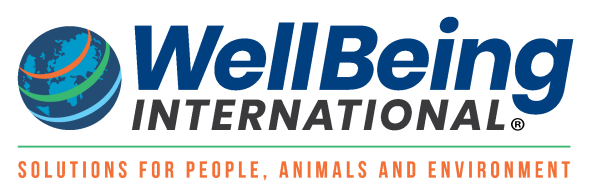




\title{
Variation in Brain Morphology of Intertidal Gobies: A Comparison of Methodologies Used to Quantitatively Assess Brain Volumes in Fish
}

\author{
Gemma E. White and Culum Brown \\ Macquarie University
}

KEYWORDS

intertidal gobies, brain morphology, histology, ellipsoid model, micro-computed tomography, neuroecology

\begin{abstract}
When correlating brain size and structure with behavioural and environmental characteristics, a range of techniques can be utilised. This study used gobiid fishes to quantitatively compare brain volumes obtained via three different methods; these included the commonly used techniques of histology and approximating brain volume to an idealised ellipsoid, and the recently established technique of X-ray micro-computed tomography (micro-CT). It was found that all three methods differed significantly from one another in their volume estimates for most brain lobes. The ellipsoid method was prone to over- or under-estimation of lobe size, histology caused shrinkage in the telencephalon, and although micro-CT methods generated the most reliable results, they were also the most expensive. Despite these differences, all methods depicted quantitatively similar relationships among the four different species for each brain lobe. Thus, all methods support the same conclusions that fishes inhabiting rock pool and sandy habitats have different patterns of brain organisation. In particular, fishes from spatially complex rock pool habitats were found to have larger telencephalons, while those from simple homogenous sandy shores had a larger optic tectum. Where possible we recommend that micro-CT be used in brain volume analyses, as it allows for measurements without destruction of the brain and fast identification and quantification of individual brain lobes, and minimises many of the biases resulting from the histology and ellipsoid methods.
\end{abstract}

\section{Introduction}

The nervous system performs a crucial role in an organism's central processing, perception and behavioural responses to external environmental stimuli. Through the progression of evolution, nervous systems have developed into a diverse array of functioning structures in a variety of shapes and sizes that few other organs can rival [Kotrschal et al., 1998]. Brain size has been shown to increase with body size among many vertebrate taxa, and after body size correlations have been taken into account, there still remains a considerable amount of variation in relative brain size. Many comparative studies across species have linked these variations in brain size to differences in a species' ecology, which suggests that 
variation in cognitive demands has resulted in the adaptive evolution of different brain sizes among species. For example, variables such as habitat complexity [Safi and Dechmann, 2005; White, 2015], social behaviour [Dunbar, 1995; Pollen et al., 2007; Gonzalez-Voyer et al., 2009a], diet [Hutcheon et al., 2002; Gonzalez-Voyer et al., 2009a], parental care strategies [Young et al., 1998], life-history traits [Iwaniuk and Nelson, 2001], mating strategy [Kolm et al., 2009] and sexual selection [Garamszegi et al., 2005; Pitnick et al., 2006] have all been shown to correlate with variation in brain size and structure in a range of vertebrate taxa.

Fish are an ideal taxonomic group for studies exploring how ecology has impacted brain evolution. The huge number of species (over 30,000 described) [Chapman, 2009] and their radiation in nearly every aquatic habitat has subjected fishes to different selection pressures unique to their niche, likely resulting in specialised sensory abilities and behaviours, which may be reflected in brain morphology [Kotrschal et al., 1998; Braithwaite, 2006; Ullmann et al., 2010]. Consequently, a vast array of brain morphologies has evolved in fishes [Kotrschal et al., 1998; Nieuwenhuys and Meek, 1998]. Although fish have developed a great diversity of brain sizes and shapes, their central nervous system still closely resembles that of other vertebrates in terms of functionality and morphology [Wullimann and Mueller, 2004; Broglio et al., 2011]. Fish brains can be divided into ten main structures that control specific cognitive functions; these include the olfactory bulbs, telencephalon, optic tectum, mesencephalon, diencephalon, pituitary, hypothalamus, cerebellum, dorsal medulla and brain stem, and many of the centres for higher processing show clear homologies with the brain regions of other vertebrates [Broglio et al., 2003; Ullmann et al., 2010]. Thus, it is not unexpected that fish should exhibit similar correlations between brain size and ecology to those that have been observed in terrestrial vertebrates. For example, studies comparing the brains of cichlids from a variety of different lakes in Africa have shown correlations between optic tectum size and diet, telencephalon and cerebellum size and structural complexity of the habitat, hypothalamus size and mating strategy, and dorsal medulla size and trophic position [Van Staaden et al., 1995; Huber et al., 1997; Pollen et al., 2007]. Despite this huge diversity, we still know comparatively little about variation in brain morphology of the vast majority of fish species.

The majority of studies exploring the links between brain size/morphology and environmental complexity in fishes have used two methods of volumetric analysis to determine brain structure size. The first method, an ellipsoid model, relies on the assumption that the volume of each brain structure can be approximated by calculating the volume of an idealised ellipsoid [Van Staaden et al., 1995; Huber et al., 1997; Wagner, 2001a, b]. This method involves obtaining linear measurements for length (I), width (W) and height $(h)$ of each brain lobe and converting them into volumetric measures using the ellipsoid formula: $0.167 \pi / w h$ [Huber et al., 1997]. The ellipsoid method has been accepted as a fast and efficient method for calculating brain volumes without the need for labour-intensive dissections, and has been used to assess a variety of brain lobe shapes [Lisney et al., 2007; Pollen et al., 2007; Burns and Rodd, 2008; Gonda et al., 2009; Kolm et al., 2009].

The second technique, known as the histological method, involves the serial sectioning of brains followed by dehydration in a graded ethanol series and staining [Kotrschal and Palzenberger, 1992; Salas et al., 1996; Vargas et al., 2000; Kihslinger and Nevitt, 2006]. Brain volume is then calculated by adding the areas of all sections and multiplying them by section thickness [Rosen and Harry, 1990; Ullmann et al., 2010]. The key advantage here is that the actual shape of each lobe can be determined and there are no underlying assumptions about approximations to ellipsoids. Although the histological method is highly laborious and time-consuming compared to the ellipsoid method, it allows the overall shape of brain lobes to be defined more clearly, makes boundaries between brain lobes more obvious, and also accounts for the presence of foliation on the brain's surface and ventricles within brain structures themselves. 
However, this method is also prone to distorting some structures more than others during the dehydration process [Ullmann et al., 2010].

More recently, methods capable of reconstructing 3D images of brain structures have been applied to the volumetric brain analyses in animals. 3D imaging techniques such as MRI (magnetic resonance imaging) and micro-CT (X-ray micro-computed tomography), unlike conventional histology, are more accurate, non-destructive, non-invasive and less time-consuming methods to image, measure and reconstruct volumes of brain structures [Metscher, 2009a; Dobrivojević et al., 2013]. Perhaps most impressive of all is that the final 3D model can be rotated and dissected along any plane using computer software. By examining the brain as a whole ex vivo, damage, deformity and shrinkage caused by histological processing can be minimised. However, high accuracy comes at a high cost (a micro-CT scanner costs AUD 87,500 to purchase and imaging time is generally AUD 90 per hour) [Metscher, 2009a].

Until recently, micro-CT was limited to imaging adipose tissues and skeletal structures in small experimental animals. In particular, in vivo imaging of the brain using micro-CT was highly challenging because contrast resolution of brain structures is much lower than that of other organs [Schambach et al., 2010]. However, newly developed techniques involving the use of X-ray absorbing radiocontrast agents have produced high-resolution micro-CT images of the brain with a spatial resolution and contrast very similar to that of micro-MRI scans [de Crespigny et al., 2008; Metscher, 2009a; Prajapati et al., 2011]. Furthermore, micro-CT scanners have proven to be easier to maintain and substantially more costeffective to run than micro-MRI scanners, which makes micro-CT more accessible for use in research laboratories where large numbers of specimens are examined [Dobrivojević et al., 2013]. Micro-CT has been used increasingly over the last 12 years for non-clinical research and more recently to assess brain volumes in honeybees [Ribi et al., 2008], Drosophila [Mizutani et al., 2007], rabbits and mice [de Crespigny et al., 2008]. These studies demonstrate that the different brain lobes are clearly defined using micro-CT imaging techniques, although individuals were not measured specifically. To the authors' knowledge, micro-CT has never been used for volumetric analyses of fish brains or to calculate brain lobe volumes.

As with all new methodologies, it is essential that they be compared to techniques already in existence (using a specific model species) so that overall accuracy and validity can be assured. Here we compare two well-established methods of brain volume assessment in fishes (ellipsoid model and histological sectioning) with a more recently developed imaging method (micro-CT). Volumes of specific brain lobes (i.e. telencephalon, optic tectum, cerebellum, hypothalamus and dorsal medulla) were measured in the following sequence using the ellipsoid model and then micro-CT or sectioning in order to determine if any volumetric differences arise between the three methods. To explore how well these techniques are able to highlight species differences in brain lobes, we used four species of intertidal gobies with already known differences in brain volumes [White, 2015] found on temperate rocky shores and sandy beaches in the Sydney region of Australia. These species were comprised of a mixture of rock pool resident species, Cocos frillgoby (Bathygobius cocosensis) and Krefft's frillgoby (Bathygobius krefftii), and sandy shore species, eastern longfin goby (Favonigobius lentiginosus) and Hoese's sandgoby (Istigobius hoesei) .

\section{Methodology}

\section{Specimen Preparation}

To examine the brain morphology of the four chosen intertidal goby species, a total of 56 fish (ca. 14 individuals of each species) were captured at low tide from a number of beaches and rock platforms in Sydney, N.S.W., Australia. Goby specimens were euthanized with an overdose of tricaine methane sulfonate (MS222), and their total length (distance from the tip of the snout on the upper jaw to the end of 
the tail) and body weight were measured. Fish specimens were immediately placed under a dissecting microscope in a bath of distilled water, and their brains were exposed dorsally by removing the top of the neurocranium (i.e. frontal, parietal and nasal bones, and in some cases the operculum). Specimens were then placed in a solution of $4 \%$ paraformaldehyde in $0.1 \mathrm{M}$ phosphate-buffered saline (PBS) buffer $\mathrm{pH} 7.2$ to fix the tissues for 2 days. After fixation was complete, we rinsed the specimens 3 times in $0.1 \mathrm{M}$ PBS buffer, waiting $15 \mathrm{~min}$ between rinses. The brains were then removed completely from the neurocranium under a dissecting microscope by severing the cranial nerves and the posterior hindbrain using fine forceps and a scalpel. We left the eyes attached to the brain to allow brains to be easily picked up and transported without being damaged. Total brain volume calculations did not include the eyes. Between imaging sessions, histological analysis and micro-CT analysis brains were stored in $0.1 \mathrm{M}$ PBS buffer at $4^{\circ} \mathrm{C}$.

\section{Volumetric Analysis Using Ellipsoid Model}

Digital images were acquired of the brains' dorsal, ventral and lateral views using a computer-assisted video image analysis system, employing an Olympus SZX16 research stereo microscope equipped with a ColourView digital camera (ColourView IIlu). Images were acquired using analySIS docu version 5.0 (Olympus Soft Imaging Solutions, 2007). Magnification on the microscope was always set between $5 \times$ and $2 \times$, and images were saved in TIF format. To reduce tissue shrinkage, all photographs were acquired while the brains were submerged in a bath of distilled water. All samples were covered by water to exactly the same depth (i.e. all specimens had exactly $1 \mathrm{~mm}$ of water over their surface) and all photographs were taken looking straight down onto the sample; therefore, the refractive index should have been exactly the same for all specimens. The ellipsoid model was used to determine the volume of each brain lobe, i.e. telencephalon, optic tectum, cerebellum, hypothalamus and dorsal medulla (fig. $1 \mathrm{a}-\mathrm{c}$ ); this model assumes that all lobes are the shape of an idealised ellipsoid [Van Staaden et al., 1995; Huber et al., 1997; Wagner, 2001a, b; Lisney and Collin, 2006; Lisney et al., 2007; Pollen et al., 2007; Ullmann et al., 2010]. Linear measurements for length ( $I)$, width ( $W$ ) and height $(h)$ of each brain lobe were acquired from digital images using analySIS docu version 5.0. The length and width of each structure was measured from a dorsal photograph, with length defined as a line parallel to the midline that extends across the maximum length of the lobe structure, and width defined as a perpendicular line extending from the midline to the lateral boundary. Lastly, height was measured from a lateral photograph, and defined as a line extending perpendicularly from the dorsal surface to the ventral surface of each lobe. Linear measures were converted into volumetric measurements $(V)$ using the formula $1 / 6 \pi / w h$ for the ellipsoid model. For examined structures that contained paired hemispheres (i.e. telencephalon, optic tectum and hypothalamus) only one hemisphere was measured and the estimated volume was doubled.

Total brain volume was also estimated using the ellipsoid formula $1 / 6 \pi / w h$, length of the whole brain was measured from the rostral edge of the telencephalon to the caudal edge of the medulla, height of the whole brain was measured from the dorsal edge of the midbrain to the ventral edge of the hypothalamus from a lateral image, and whole brain width was measured across the two optic tecta from a dorsal image.

\section{Micro-CT Acquisition and Volumetric Analysis}

Four brains for each of the four fish species were used in micro-CT acquisition. Prior to imaging, the fish brains were stained in $2 \%$ osmium tetroxide for 2 days to aid in visualisation of brain structures. Micro-CT employs a microfocus X-ray source operating in the cone-beam method to create a visual image of the surface and internal structures of the brain in three dimensions, without destructive or extensive specimen preparation (see [ACMM, 2011]). Brain samples were suspended in a clear pipette tip attached to a plastic stub with plasticine and examined with a Skyscan 1172 Xray micrograph version 1.5 (Belgium, 2009) fitted with a Hamamatsu 10-megapixel camera. Projection images were obtained at $59 \mathrm{kV}$ and 167 

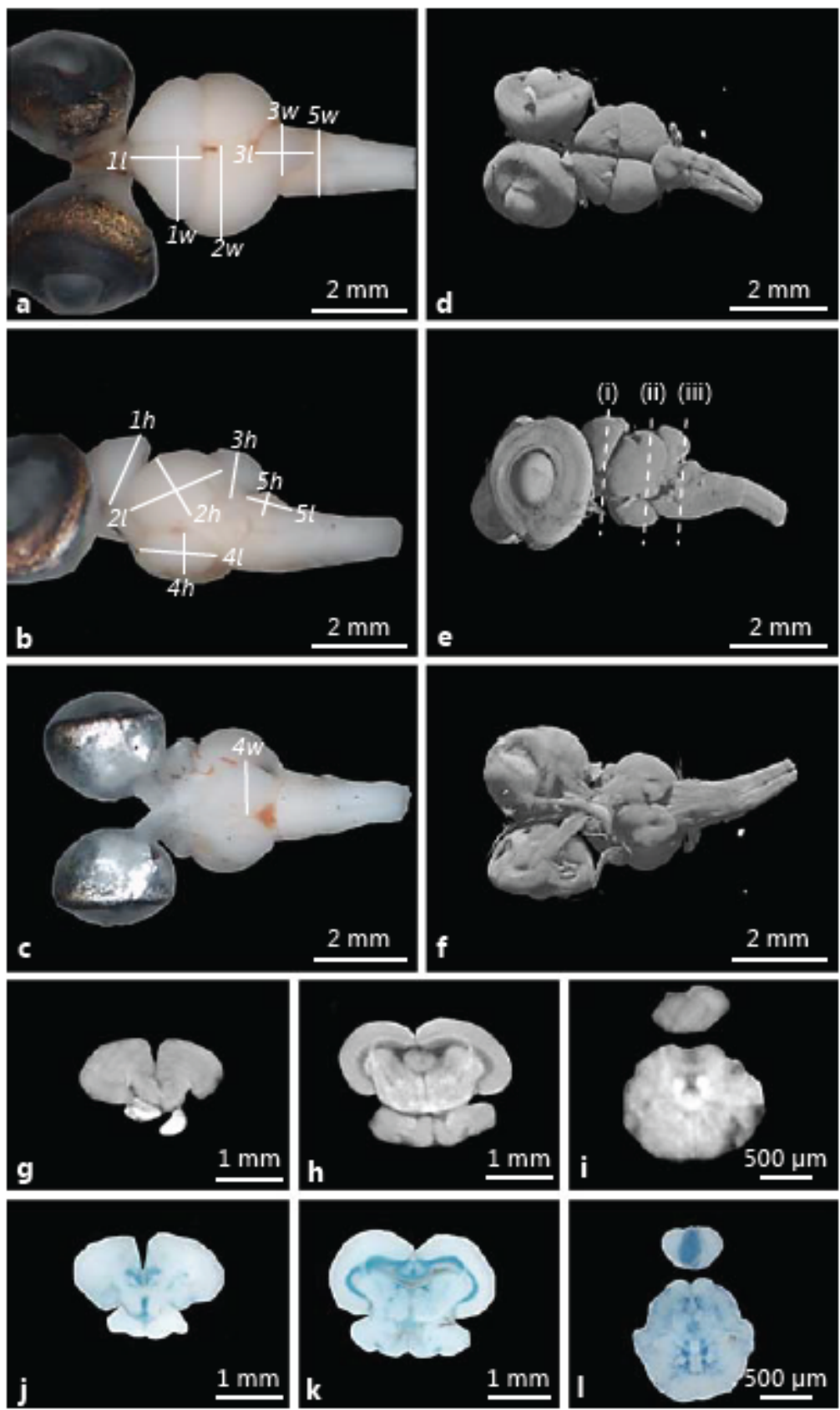

Fig. 1. Illustration of the 3 methods used to assess brain volumes in the Cocos frillgoby Bathygobius cocosensis : ellipsoid method ( $a-c$ ), micro-CT method ( $d-i$ ) and histological sectioning method ( $j-1$ ). Key to abbreviations in $\mathrm{a}-\mathrm{c}: \mathbf{1}=$ telencephalon, $2=$ optic tectum, $3=$ cerebellum, $4=$ hypothalamus, $5=$ dorsal medulla. The dashed line (i) indicates the approximate position of the transverse sections $g$ and $j$ that pass through the telencephalon, the dashed line (ii) indicates the approximate position of the transverse sections $h$ and $k$ that pass through the optic tectum, cerebellum and hypothalamus, and the dashed line (iii) indicates the approximate position of the transverse sections $i$ and $I$ that pass through the cerebellum and dorsal medulla. In the ellipsoid method, the length (I), width $(w)$ and height $(h)$ were measured from photographs using analySIS docu. In the micro-CT method, the brain was scanned producing a stack of reconstructed 2D cross-sectional images and the boundaries of the brain lobes were measured using analysis docu. In the histology method, the brain was stained and sectioned, and the boundaries of the brain lobes measured using analysis docu. 
$\mu \mathrm{A}\left(\right.$ pixel size $=9.36 \mu \mathrm{m}$ for $I, w$ and $h$ ). The specimen was then slowly rotated $360^{\circ}$ and a sequence of images were 'back projected' onto an X-ray-sensitive detector [Hsieh, 2003; Kalender, 2005]. A rotation of $0.39^{\circ}$ was used between each image acquisition, providing a series of 925 projection images in 45 min. From this series of images, a stack of 2D virtual sections was reconstructed for each brain sample. 3D modelling and analysis reconstruction of the brain were obtained with NRecon software (version: 1.6.6; fig. $1 \mathrm{~d}-\mathrm{f}$ ). This program allows the reconstruction of objects from the stack of 2D virtual sections after segmentation by aligning cross-sectional images to the rotational axis of the specimen and displays them as a 3D matrix of brightness values [Metscher, 2009a]. Reconstructed 3D models were obtained using VGStudioMax software version 1.2.1 (Germany, 2006).

Only one 2D acquisition slice out of every 11 slices (84 slices in total) was used for volumetric analysis (fig. $1 \mathrm{~g}-\mathrm{i}$ ). The cross-sectional area of each brain lobe was traced digitally on each virtual slice using analySIS docu version 5.0. This program counted the number of pixels contained within the drawn polygon. We then calculated the total volume of each lobe by adding the number of pixels from all traced slices and multiplying them by the size of a single pixel $(9.3 \times 9.3 \mu \mathrm{m})$ and by the slice thickness $(102.3$ $\mu \mathrm{m})$. Brain lobe structural boundaries used in this study closely followed published descriptions by Davis and Northcutt [1983] and Wullimann et al. [1996]. For examined structures that contained paired hemispheres (i.e. telencephalon, optic tectum and hypothalamus), only one hemisphere was measured and the estimated volume was doubled. The entire brain was also traced digitally from virtual slices and total brain volume was calculated by adding the areas from all traced slices and multiplying them by the section thickness.

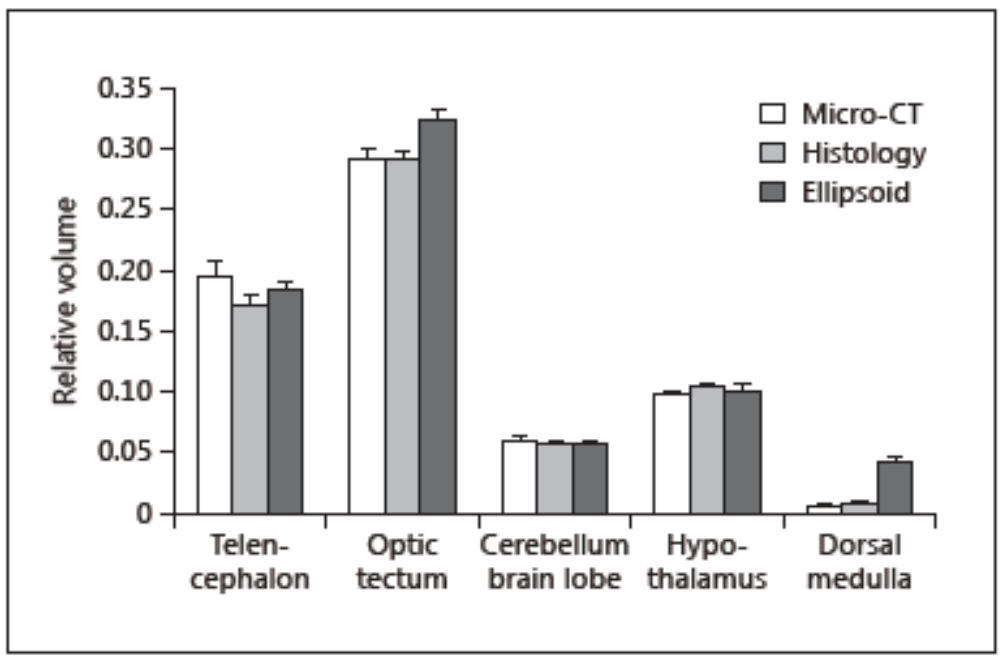

Fig. 2. Quantitative assessment (means $\pm S E$ ) of brain lobe volumes compared using the ellipsoid method, histology and micro-CT.

\section{Histology and Volumetric Analysis}

Ten brains from each of the four fish species were prepared for sectioning following calculation of brain volumes using the ellipsoid method; this involved staining brains in a solution of $1 \%$ methylene blue, $0.6 \%$ sodium bicarbonate and $40 \%$ glycerol (diluted with water to a 1:1 ratio) for 2 min, embedding them in agarose and storing them overnight in $0.1 \mathrm{M}$ PBS buffer at $4^{\circ} \mathrm{C}$. Brains were sliced into a series of $100-$ $\mu \mathrm{m}$-thick sections on a Leica VT 1000S Vibratome and mounted on a microscope slide (4 per slide) or stored in a well plate containing 0.1 M PBS to avoid dehydration until the imaging was complete. Sections 
were photographed immediately using a digital camera (ColourView IIlu) attached to an Olympus SZX16 Research Stereo Microscope (fig. $1 \mathrm{j}-1$ ). The cross-sectional area of each brain lobe was traced digitally and analysed using analySIS docu version 5.0. The total volume of each lobe was then calculated by adding the areas from all traced sections and multiplying them by the section thickness $(100 \mu \mathrm{m})$ [Rosen and Harry, 1990; Costa et al., 2011]. For examined structures that contained paired hemispheres (i.e. telencephalon, optic tectum and hypothalamus), only one hemisphere was measured and the estimated volume was doubled. The entire brain was also traced digitally from sections and total brain volume was calculated by adding the areas from all traced sections and multiplying them by the section thickness.

\section{Statistical Analysis}

To compare the relative volume estimates between the three methodologies and goby species, repeated measures ANOVA was performed on ellipsoid and histology methods and then on the ellipsoid and microCT with species included as a factor in each analysis. We were unable to include all methodologies in a single repeated measures analysis because although we have ellipsoid data for all fish, we do not have data for the same fish for the histology and micro-CT methods. Micro-CT staining technique dyes brain tissues black and makes lobe boundaries difficult to identify for histological analysis, and a brain cannot be reassembled for micro-CT scanning once it has been sectioned for histology. Thus, to compare histology and micro-CT methodologies, we used an additional one-way ANOVA test with species included as a factor.

Brain volume comparisons can be complicated by the fact that brain size exhibits an allometric relationship with body size [Striedter, 2005; Deaner et al., 2007; Gonzalez-Voyer et al., 2009a, b]. To control for these allometric effects, we calculated the relative lobe volume for each fish specimen [Bullmore et al., 1995; Burish et al., 2004; Wiper et al., 2014]. For example, when examining relative lobe volume, body size was controlled for by dividing lobe volume by total brain volume to give the relative value (i.e. telencephalon volume/total brain volume $=$ relative telencephalon volume). Furthermore, all fish specimens used in this study were large $(>4 \mathrm{~cm})$ to ensure that variation in brain/body size was limited between specimens within the same species. However, we cannot rule out the possibility that relative size of different brain regions may vary with age, even if animals have the same body length. All statistical analyses were performed using IBM SPSS statistics version 20 (IBM Corp. 2011) and StatView version 5.0.1 (SAS Institute Inc., 1998).

\section{Results}

\section{Method Comparison}

All three methods differed significantly from one another for all brain lobes except for the hypothalamus and cerebellum (fig. 2). In the hypothalamus, similar volumes were obtained using ellipsoid, histology and micro-CT methods. However, histology was found to generate significantly larger volume estimates than micro-CT $\left(F_{1,48}=8.0786, p=0.007\right)$. The hypothalamus is shaped very much like an idealised ellipsoid. All three methods were found to generate very similar volume estimates when analysing the cerebellum ( $p>0.05$ in all cases). However, micro-CT was found to generate marginally larger volume estimates than histology $\left(F_{1,48}=3.038, p=0.087\right)$. The cerebellum has a tear drop shape; the tail end, known as the valvula cerebellum, cannot be viewed in surface photographs acquired for use in the ellipsoid method because it is hidden beneath the optic tectum.

Significant differences were found between all three methods for the telencephalon and dorsal medulla ( $p<0.009$ in all cases). For the telencephalon, quantitative assessment using the micro-CT method resulted in the largest volume followed by the ellipsoid method and finally histology (table 1). The 
telencephalon is shaped very much like an ellipsoid. For the dorsal medulla, the idealized ellipsoid yielded the largest volume, followed by histology and finally micro-CT (fig. 2). The dorsal medulla has a crescentlike shape that cannot be seen using the ellipsoid method.

Table 1. Multivariate ANOVA method contrast results for five brain lobes

\begin{tabular}{|c|c|c|c|c|c|c|c|c|c|}
\hline & \multicolumn{3}{|c|}{ Ellipsoid vs. histology } & \multicolumn{3}{|c|}{ Ellipsoid vs. micro-CT } & \multicolumn{3}{|c|}{ Histology vs. micro-CT } \\
\hline & d.f. & F value & $p$ value & d.f. & F value & $p$ value & d.f. & F value & $p$ value \\
\hline Telencephalon & 36 & 8.912 & $<0.0051(E)$ & 12 & 9.562 & $0.0092(\mathrm{M})$ & 48 & 16.511 & $0.0002(\mathrm{M})$ \\
\hline Optic tectum & 36 & 12.284 & $0.0012(E)$ & 12 & 7.641 & $0.0171(E)$ & 48 & 0.078 & $0.7808(-)$ \\
\hline Hypothalamus & 36 & 2.12 & $0.154(-)$ & 12 & 0.12 & $0.735(-)$ & 48 & 8.08 & $0.007(\mathrm{H})$ \\
\hline Cerebellum & 36 & 1.216 & $0.2774(-)$ & 12 & 0.245 & $0.6298(-)$ & 48 & 3.039 & $0.087(-M)$ \\
\hline Dorsal medulla & 36 & 312.88 & $<0.0001(E)$ & 12 & 150.54 & $<0.0001(E)$ & 48 & 15.055 & $0.0003(H)$ \\
\hline
\end{tabular}

Table 2. Multivariate ANOVA species contrast results for the five brain lobes

\begin{tabular}{|c|c|c|c|c|c|c|c|c|c|}
\hline & \multicolumn{3}{|c|}{ Ellipsoid vs. histology } & \multicolumn{3}{|c|}{ Ellipsoid vs. micro-CT } & \multicolumn{3}{|c|}{ Histology vs. micro-CT } \\
\hline & d.f. & $F$ value & $p$ value & d.f. & F value & $p$ value & d.f. & F value & $p$ value \\
\hline Telencephalon & 36 & 22.692 & $<0.0001(\mathrm{R}, \mathrm{C})$ & 12 & 25.251 & $<0.0001(\mathrm{R}, \mathrm{C})$ & 48 & 55.162 & $<0.0001(\mathrm{R}, \mathrm{C})$ \\
\hline Optic tectum & 36 & 6.6284 & $0.0011(\mathrm{~S}, \mathrm{H})$ & 12 & 3.457 & $0.0513(\mathrm{~S}, \mathrm{H})$ & 48 & 35.237 & $<0.0001(\mathrm{~S}, \mathrm{H})$ \\
\hline Hypothalamus & 36 & 1.985 & $0.134(-)$ & 12 & 3.812 & $0.0395(-, \mathrm{K})$ & 48 & 7.36 & $0.004(-, \mathrm{H})$ \\
\hline Cerebellum & 36 & 16.932 & $<0.0001(-, \mathrm{C})$ & 12 & 4.082 & $0.0327(-, C)$ & 48 & 10.282 & $<0.0001(-, C)$ \\
\hline Dorsal medulla & 36 & 1.191 & $0.327(-)$ & 12 & 2.174 & $<0.144(-)$ & 48 & 6.047 & $0.0014(-, \mathrm{K})$ \\
\hline
\end{tabular}

Within the optic tectum, no significant differences in relative volumes were observed between the histology and micro-CT methods ( $p>0.05$ ). However, the ellipsoid method was found to generate larger volume estimates than both histology and micro-CT when analysing the optic tectum $(p<0.01$ in both cases; table 1). The optic tectum has a crescent-like shape that cannot be viewed in surface photographs acquired for use in ellipsoid method.

All three methods generated clear differences between the four species examined here (table 2). In particular all methods indicated that the telencephalon was significantly larger for species inhabiting rock pools (Cocos frillgoby and Krefft's goby) than those inhabiting sandy shores (eastern longfin goby and Hoese's goby; $p<0.0001$; fig. 3 a). Furthermore, all methods demonstrated that the optic tectum was significantly larger in sandy shore species (eastern longfin goby and Hoese's goby) than species inhabiting rock pools (Cocos frillgoby and Krefft's goby; $p<0.05$; fig. 3 b).

\section{Discussion}

Brain size and structure have been correlated with spatial complexity within a habitat, dietary requirements and a range of social behaviours in comparative studies on a range of taxa, including primates [Barton, 1996; Reader and Laland, 2002], birds [Healy and Guilford, 1990; Clayton, 2001], 
ungulates [Shultz and Dunbar, 2006] and more recently fishes [Pollen et al., 2007; Gonzalez-Voyer and Kolm, 2010; Wilson and McLaughlin, 2010; Costa et al., 2011; Kotrschal et al., 2012a, b; Lecchini et al., 2014; Wiper et al., 2014]. These correlations were generated by determining brain volumes using the ellipsoid method and histology, and this study has added a further method for consideration, micro-CT. Although each of the three methods has its own benefits, this study reveals they each also have clear drawbacks; thus, there is no best methodology that can be applied to the assessment of brain volumes. Time, money, accuracy and precision are all important variables to consider when deciding which method to employ. If budget is of no concern, however, then micro-CT is arguably the most accurate methodology because it avoids many of the biases that are associated with the histology and ellipsoid methods. Our results also indicate that it is wise for future studies to use caution when utilising any method to assess volume individually or in combination with other methods.

A large number of comparative studies have made use of the ellipsoid method to determine brain volumes in fishes [Van Staaden et al., 1995; Huber et al., 1997; Lisney and Collin, 2006; Pollen et al., 2007; Burns and Rodd, 2008; Gonzalez-Voyer et al., 2009b; Kolm et al., 2009; Kotrschal et al., 2012a], largely due to the nature of fish brain lobes to form elliptical shapes. However, the ellipsoid method is less useful in calculating the brain lobe volume in terrestrial vertebrates as their brain lobes do not conform to the standard ellipsoid shape required for this method to work accurately. When compared to the specialised machines required for micro-CT and MRI, or the labour-intensive and time-consuming nature of histology, the ellipsoid method is not only highly efficient in terms of time investment but also requires only simple, readily available equipment (table 3). This allows a greater quantity of specimens to be examined, which is a necessary requirement in experimental protocols that aim to verify correlations between ecological or social forces and specific brain lobes. The ellipsoid method operates on one main assumption, which is that all brain lobes resemble an ellipsoid in shape and thus volumes can be estimated using a simple formula. However, this assumption may cause inaccuracies in the results as the ellipsoid method does not account for changes in lobe shape such as variation in concavity within a single structure (i.e. cichlids) [Pollen et al., 2007], foliation or external folding in lobe structure (i.e. sharks) [Lisney and Collin, 2006], the presence of a sulcus ypsiloniformis splitting the lobe (i.e. barramundi) [Ullmann et al., 2010] or the crescent-like shape of the optic tectum observed in this study. A method such as this may also create problems if there were considerable interspecies variations in the shape of brain lobes. In this study, however, we compared brains of four closely related species where there were no major changes in lobe shapes. Although the ellipsoid method overestimated the volumes of the whole brain, optic tectum and dorsal medulla, and underestimated the volume of the cerebellum, we found that it nevertheless obtained consistent estimates of brain lobe volumes within the gobiid group. The whole brain was overestimated because it does not form a smooth convex ellipsoid, but it includes quick changes in curvature between lobe structures and also contains areas that are concave shaped. The optic tectum was likely overestimated because the method included other midbrain structures (e.g. torus semicircularis, mesencephalic tegmentum, etc.), while the sectioning method consisted only of the optic tectum. For brain lobes such as the hypothalamus and cerebellum, which appear to mimic the smooth convex shape of ellipsoids quite closely, the ellipsoid method is likely to provide relatively accurate volume estimates. This was validated by the similar volume estimates that were obtained using the ellipsoid, histology and micro-CT methods ( $p>0.05)$. A study by Ullmann et al. [2010] looking at barramundi brain volumes found that the olfactory bulbs closely mimic the shape of idealised ellipsoids, while the shape of the telencephalon and optic tectum deviated from the elliptical shape and thus was overestimated by the ellipsoid method.

Histology is a long-established method for calculating brain volumes and a considerable number of studies have utilised varying stereological techniques [Haug, 1986; Rosen and Harry, 1990; White, 2015]. However, histology is labour-intensive, time-consuming and provides only 2D views of brain areas (table 
3). The majority of histological studies commonly involve staining, dehydration and clearing of brain sections, which allows for enhanced contrast and visualisation of larger numbers of neurons [Kotrschal and Junger, 1988; Kotrschal and Palzenberger, 1992; Kihslinger et al., 2006; Kihslinger and Nevitt, 2006; Ayari et al., 2012]. However, the process of dehydration has been demonstrated to result in tissue shrinkage of up to $70 \%$ and also geometric distortions [Grace and Llinas, 1985; de Crespigny et al., 2008; Ullmann et al., 2010]. In this study our histology methods did not involve any form of dehydration; in fact, brains were always submerged in a solution of PBS before, during and after sectioning and imaging to reduce shrinkage. We found that the histology method predicted volumes that were consistent with microCT-generated volumes for all brain lobes except the telencephalon, which obtained a smaller volume than both the micro-CT and ellipsoid methods. Previous comparative studies that have used point counting to generate volumes of MRI virtual sections and untreated physical (histological) sections also observed no significant differences between the two methods [Garcia-Finana et al., 2003; Jelsing et al., 2005]. However, Ullmann et al. [2010] found that the olfactory bulbs, optic tectum and telencephalon shrink to varying degrees when using the histological method to generate volume estimates.

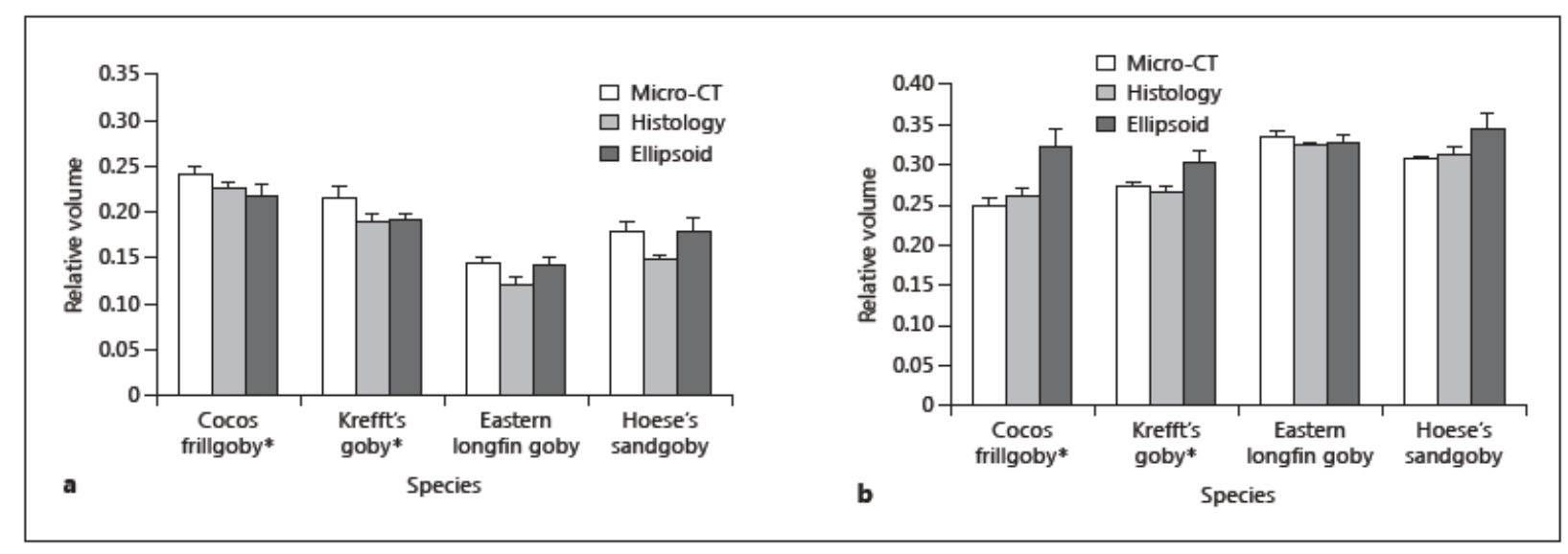

Fig. 3. Quantitative assessment (means $\pm \mathrm{SE}$ ) of brain lobe volumes compared using the ellipsoid method, histology and micro-CT in four goby species with known brain lobe differences, exploring relative investment in neural tissue in the telencephalon (a) and optic tectum (b). Species denoted with an asterisk are rock pool residents, those without are sandy shore residents.

High-field small-bore MRI scanners are expensive to purchase and run (equipment costs millions of dollars to buy and thousands of dollars to run), involve a considerable amount of skill and effort of specifically trained operators for parameter settings, and require facilities suitable for high magnetic fields [Ullmann et al., 2010; Hayasaka et al., 2012; Dobrivojević et al., 2013]. In contrast, micro-CT provides non-destructive, high-resolution in vivo and ex vivo images of brain structures, with rapid data acquisition capabilities and spatial resolution and contrast very similar to that of MRI scans [de Crespigny et al., 2008; Prajapati et al., 2011]. Micro-CT scanners also have the added advantage of being considerably cheaper and relatively simple to use and maintain compared to MRI. Furthermore, micro-CT scanners do not have any installation restrictions or requirements for a radiation shield, thus making them more readily available for use in research laboratories as opposed to MRI scanners [Dobrivojević et al., 2013] (table 3). The micro-CT method has most often been employed to image a range of mineralised animal tissues (reviewed in Neues and Epple [2008]); however, its widespread usage in comparative morphology has been limited due to the low intrinsic X-ray contrast of non-mineralised tissues. Through the recent development of techniques using X-ray contrast enhancement stains (reviewed in Metscher [2009a]), 
micro-CT imaging of soft tissues in animal samples is now possible. Osmium tetroxide is the most commonly used contrast stain for micro-CT soft tissue imaging and was used in this study; it has electronbinding energies that promote strong X-ray absorption, is available to most research laboratories and can easily bind to cell membranes, nerves and other lipid-rich structures. However, osmium is highly toxic, has a slow penetration rate, is costly to purchase and dispose of, and has poor staining potential for samples that have been stored in ethanol [Metscher, 2009a]. It is important to note that other less toxic and lower priced contrast stains such as inorganic iodine, phosphotungstic acid and phosphomolybdic acid can also provide good contrast [Johnson et al., 2006; de Crespigny et al., 2008; Metscher, 2009a] equal to that of osmium tetroxide [Degenhardt et al., 2010]. However, they also have their own drawbacks; phosphotungstic acid has a slow tissue penetration rate (24 $\mathrm{h}$ or more) and inorganic iodine may overstain some mineralised tissues causing variations in contrast intensity and permanence in different sample preparations [Metscher, 2009b]. Moreover, when using specialised imaging protocols and contrast stains, many of the biases generated by ellipsoid and histology methods can be eliminated. Micro-CT imaging allows the brain to be virtually sectioned on any plane, so internal and external brain structures can be viewed and accurately quantified, which in turn nullifies any biases resulting from inaccurate assumptions made about lobe shapes by the ellipsoid method. Furthermore, the use of contrast stains such as osmium appears to eliminate much of the sample shrinkage that occurs when using histological methods [Metscher, 2009b]. Much like this study, Ullmann et al. [2010] found that 3D imaging techniques, in the form of MRI, also avoided many of the problems associated with histology and the ellipsoid methods, i.e. shrinkage and overestimation.

Table 3. Approximate time and money spent per specimen for each of the three methods

\begin{tabular}{|c|c|c|}
\hline Method & Time & Cost \\
\hline Ellipsoid & $\begin{array}{l}\text { Image capture = } 30 \mathrm{~min} \\
\text { measurements }=10 \mathrm{~min}\end{array}$ & $\begin{array}{l}\text { Olympus SZX16 Microscope purchase price = AUD 12,000 - } \\
35,000 ; \text { consumables = AUD 1; labour = AUD } 27\end{array}$ \\
\hline $\begin{array}{l}\text { Histology } \\
\text { (as performed } \\
\text { in this study) }\end{array}$ & $\begin{array}{l}\text { Staining }=2 \mathrm{~min} ; \text { embedding in agarose }=12 \mathrm{~h} ; \\
\text { vibratome }(\text { sectioning })=40 \text { min; } \\
\text { imaging }=40 \text { min; measurements }=1 \mathrm{~h}\end{array}$ & $\begin{array}{l}\text { Olympus SZX16 Microscope purchase price = } \\
\text { AUD 12,000 - 35,000; consumables = AUD 10; } \\
\text { labour = AUD } 100\end{array}$ \\
\hline $\begin{array}{l}\text { Histology } \\
\text { (with ethanol } \\
\text { dehydration) }\end{array}$ & $\begin{array}{l}\text { Cryoprotected in sucrose = } 2 \mathrm{~h} \text {; } \\
\text { embedding in OCT compound = } 12 \mathrm{~h} \text {; } \\
\text { cryostat (sectioning) = 40 min; staining = } 4-15 \mathrm{~min} ; \\
\text { dehydration }(70,70,100,100 \text { and } 100 \% \text { ethanol })=5 \mathrm{~h} \text {; } \\
\text { clearing }(100 \% \text { xylene } .3)=3 \mathrm{~h} ; \\
\text { imaging = } 40 \text { min; measurements }=1 \mathrm{~h}\end{array}$ & $\begin{array}{l}\text { Olympus SZX16 Microscope purchase price = } \\
\text { AUD 12,000 - 35,000; consumables = AUD 30; } \\
\text { labour = AUD 535 }\end{array}$ \\
\hline MRI & $\begin{array}{l}\text { Staining = } 10 \mathrm{~min}-120 \mathrm{~h} \text { (depending on stain used); } \\
\text { scanning = } 14 \mathrm{~h} \text {; manual measurements }=1 \mathrm{~h} 30 \mathrm{~min}\end{array}$ & $\begin{array}{l}\text { MRI scanner purchase price = AUD } 600,000-1,500,000 \\
\text { scan cost and consumables = AUD 300; labour = AUD } 80\end{array}$ \\
\hline $\begin{array}{l}\text { Times do not } \\
48 \text { h respectiv } \\
\text { Ullmann. Micr } \\
\text { and convertec } \\
\text { olympus-lifesc }\end{array}$ & $\begin{array}{l}\text { le brain extraction or fixation processing as this was of ec } \\
\text { Information on MRI time was acquired from Ullmann et a } \\
\text { and MRI scanner purchase prices were obtained from ht } \\
\text { UD, and Olympus SZX16 Microscope purchase prices we } \\
\text { e.com/en/microscopes/stereo/. Labour was calculated at }\end{array}$ & $\begin{array}{l}\text { duration regardless of the method used ( } 30 \text { min and } \\
10] \text { and personal communications with Jeremy F.P. } \\
\text { ffers. blockimaging.com/imaging-equipment-salesprices } \\
\text { btained from customer enquiries at http://www. } \\
40 \text { per hour. }\end{array}$ \\
\hline
\end{tabular}


Despite the clear advantages, micro-CT still possesses a number of limitations that may have an impact on its use in the near future. For example, contrast on micro-CT images can be a limiting factor in comparative neuromorphological studies because any volumes produced via this method depend largely on the ability of the observer to detect the boundaries of the brain area being measured. Since micro-CT is still only a recently established technique in small-animal imaging, obtaining the most useful set of scanner parameters (e.g. scanning time, filters, X-ray energy and wavelength) for different types of specimens and developing a contrast stain that will highlight the specific tissues needed for investigation can cause issues for micro-CT users [Bartling et al., 2007; Metscher, 2009a, b; Paterson et al., 2014]. If system settings and contrast stains are not ideal, then micro-CT images may exhibit poor tissue contrast (i.e. shading), creating difficulties for observers in distinguishing tissue boundaries and resulting in biased volumetric calculations. Poor image contrast may account for micro-CT obtaining a lower dorsal medulla volume estimate than histology and ellipsoid methods in this study. Furthermore, if there is more than one person examining samples, then there may also be observer-dependent interpretation of images, resulting in intra-observer bias. However, we reduced these biases in the present study by allowing only one observer to perform volumetric assessments, and by only measuring brain lobes with clearly defined boundaries on both micro-CT images and also in published brain atlases.

Despite the differences noted in volume estimates between the three methods, all methods depict qualitatively similar variation in lobe volumes between the four goby species studied here. All methods indicated that species inhabiting rock pools (Cocos frillgoby and Krefft's goby) had larger telencephalons, while species inhabiting sandy shores (eastern longfin goby and Hoese's goby) had a larger optic tectum. Thus, all methods provide support for the theory that various fish species trade off neural investment in specific brain lobes depending on the environment in which they live. These data suggest that rock pool species require greater spatial learning abilities to navigate in their spatially complex environment, while sand-dwelling species, on the other hand, likely have reduced need for spatial learning due to their spatially simple habitat and a greater need for visual acuity (for further detail on brain lobe differences between species, see [White, 2015]). Therefore, it seems that for the species studied here - regardless of the method used - there is a guarantee to obtain fairly precise estimates of volume differences between the species for comparative purposes even if the volume estimates are not entirely accurate. The similarity in brain lobe structure for each species is probably the key. However, this may not be true in other fishes or animal families. For example, previous studies have observed that brain shrinkage can vary between species [Weil, 1928] and also differing stages of development, with the brains of younger specimens shrinking to a greater extent than those of older specimens [Haug, 1986; Uylings et al., 1986]. While we believe this did not affect the results of this study, as we assessed individuals of a similar size and age, other studies have been known to analyse a range of species at unknown or varying stages of development [Kotrschal and Junger, 1988], which may be unintentionally biasing any potential conclusions they make.

\section{Conclusions}

A number of previous studies on other taxa have questioned the accuracy of some of the volume assessment methods currently in use [Iwaniuk and Nelson, 2002; Healy and Rowe, 2006; LaDage et al., 2009; Ullmann et al., 2010]. We would also advise that it is wise to use caution when interpreting data collected on brain volumes (across studies and techniques) until measures of the size of relative brain lobes become more accurate. Future comparative studies should ensure that (i) methods for volumetric analysis are chosen for their accuracy (i.e. no shrinkage or overestimentation) rather than efficiency, (ii) lobe boundaries are defined using established literature, (iii) methods known to cause lower levels of tissue shrinkage should be utilised if histology is used to process samples and (iv) brain tissues are fresh or preserved using the same fixative, as fixation has been shown to cause changes in tissue structure 
[Purea and Webb, 2006; Kim et al., 2009; Ullmann, 2010] and different fixatives will have different effects on tissue volumes [Stowell, 1941; Bahr et al., 1957].

Finally, although all three methods of brain volume assessment studied here allowed the same conclusions to be drawn when comparing different species, it should be noted that micro-CT allowed for measurements without destruction of the brain, with fast identification and quantification of individual lobes, and a minimisation of many of the biases resulting from the histology and ellipsoid methods. This all indicates that micro-CT is a highly effective machine for fast, uncomplicated, non-invasive, and economical imaging of fish brains for volumetric analyses. Thus, where possible, micro-CT should be considered for use in comparative volumetric studies.

\section{Acknowledgements}

Many thanks are due to the Macquarie University Microscopy Unit for use of facilities and technical assistance from staff. The authors would also like to thank the AMMRF (Australian Microscopy \& Microanalysis Research Facility) at the Electron Microscope Unit, University of Sydney, for use of the Skyscan 1172 Micro-CT scanner as well as scientific and technical assistance provided by staff. This project was funded by Macquarie University. Experimental methods in this study conformed to the standards set by Macquarie University Animal Ethics committee (ARA No. 2011/021).

\section{References}

ACMM, Sydney University (2011): X-RAY Facilities: Skyscan 1072 Micro-Computed Tomography. http://sydney.edu.au/acmm/facilities/xray/index.shtml (accessed August 14, 2011).

Ayari B, Landoulsi A, Soussi-Yanicostas N (2012): Localization and characterization of kal 1.a and kal 1.b in the brain of adult zebrafish (Danio rerio). Brain Res Bull 88: 345-353.

Bahr GF, Bloom G, Friberg U (1957): Volume changes of tissue in physiological fluids during fixation in osmium tetroxide or formaldehyde and during subsequent treatment. Exp Cell Res 12: 342-355.

Bartling SH, Stiller W, Semmler W, Kiessling F (2007): Small animal computed tomography imaging. Curr Med Imaging Rev 3: 45-59.

Barton RA (1996): Neocortex size and behavioral ecology in primates. Proc Biol Sci 263: 173-177.

Braithwaite VA (2006): Cognitive ability in fish; in Sloman KA, Wilson RW, Balshine S (eds): Fish Physiology: Behaviour and Physiology of Fish. Amsterdam, Elsevier, vol 24, pp 1-37.

Broglio C, Gomez A, Duran E, Salas C, Rodriguez F (2011): Brain and cognition in teleost fish; in Brown C, Laland K, Krause J (eds): Fish Cognition and Behavior, ed 2. Oxford, Wiley-Blackwell, pp 325358.

Broglio C, Rodriguez F, Salas C (2003): Spatial cognition and its neural basis in teleost fishes. Fish Fish 4: 247-255.

Bullmore E, Brammer M, Harvey I, Ron M (1995): Against the laterality index as a measure of cerebral asymmetry. Psychiatry Res 61: 121-124.

Burish MJ, Kueh HY, Wang SH (2004): Brain architecture and social complexity in modern and ancient birds. Brain Behav Evol 63: 107-124.

Burns JG, Rodd FH (2008): Hastiness, brain size and predation regime affect the performance of wild guppies in a spatial memory task. Anim Behav 76: 911-922. 
Chapman AD (2009): Numbers of Living Species in Australia and the World. A Report for the Australian Biological Resources Study, Australian Biodiversity Information Services, Toowoomba, Australia. http://www.environment.gov.au/node/13866 (accessed May 24, 2014).

Clayton NS (2001): Hippocampal growth and maintenance depend on food-catching experience in juvenile mountain chickadees (Poecile gambeli) . Behav Neurosci 115: 614-625.

Costa SS, Andrade R, Carneiro LA, Goncalves EJ, Kotrschal K, Oliveira RF (2011): Sex differences in the dorsolateral telencephalon correlate with home range size in blenniid fish. Brain Behav Evol 77: 55-64.

Davis RE, Northcutt RG (1983): Fish Neurobiology. Ann Arbor, University of Michigan Press.

Deaner RO, Isler K, Burkart J, van Schaik C (2007): Overall brain size, and not encephalization quotient, best predicts cognitive ability across non-human primates. Brain Behav Evol 70:115-124.

de Crespigny A, Bou-Reslan H, Nishimura MC, Phillips H, Carano RA, D'Arceuil HE (2008): 3D micro-CT imaging of the postmortem brain. J Neurosci Methods 171: 207-213.

Degenhardt K, Wright AC, Horng D, Padmanabhan A, Epstein JA (2010): Rapid 3D phenotyping of cardiovascular development in mouse embryos by micro-CT with iodine staining. Circ Cardiovasc Imaging 3: 314-322.

Dobrivojević M, Bohaček I, Erjavec I, Gorup D, Gajović S (2013): Computed microtomography visualization and quantification of mouse ischemic brain lesion by nonionic radio contrast agents. Croat Med J 54: 3-11.

Dunbar RIM (1995): Neocortex size and group size in primates: a test of the hypothesis. J Hum Evol 28 : 287-296.

Garamszegi LZ, Eens M, Erritzo J, Moller AP (2005): Sperm competition and sexually size dimorphic brains in birds. Proc Biol Sci 272: 159-166.

Garcia-Finana M, Cruz-Orive LM, Mackay CE, Pakkenberg B, Roberts N (2003): Comparison of MR imaging against physical sectioning to estimate the volume of human cerebral compartments. Neuroimage 18: 505-516.

Gonda A, Herczeg G, Merila J (2009): Habitat-dependent and -independent plastic responses to social environment in the nine-spined stickleback (Pungitius pungitius) brain. Proc Biol Sci 276: 161167.

Gonzalez-Voyer A, Winberg S, Kolm N (2009a): Social fishes and single mothers: brain evolution in African cichlids. Proc Biol Sci 276: 161-167.

Gonzalez-Voyer A, Winberg S, Kolm N (2009b): Brain structure evolution in a basal vertebrate clade: evidence from phylogenetic comparative analysis of cichlid fishes. BMC Evol Biol 9: 238.

Gonzalez-Voyer A, Kolm N (2010): Sex, ecology and the brain: evolutionary correlates of brain structure volumes in Tanganyikan cichlids. PLoS One 5:e14355.

Grace AA, Llinas R (1985): Morphological artifacts induced in intracellularly stained neurons by dehydration: circumvention using rapid dimethyl sulfoxide clearing. Neuroscience 16: 461-475.

Haug H (1986): History of neuromorphometry. J Neurosci Methods 12: 1-17.

Hayasaka N, Nagai N, Kawao N, Niwa A, Yoshioka Y, et al. (2012): In vivo diagnostic imaging using micro-CT: sequential and comparative evaluation of rodent models for hepatic/brain ischemia and stroke. PLoS One 7:e32342.

Healy S, Guilford T (1990): Olfactory-bulb size and nocturnality in birds. Evolution 44: 339-346.

Healy SD, Rowe C (2006): A critique of comparative studies of brain size. Proc Biol Sci 274: 453-464.

Hsieh J (2003): Computed Tomography - Principles, Design, Artifacts, and Recent Advances. Bellingham, SPIE Press.

Huber R, Van Staaden MJ, Kaufman LS, Liem KF (1997): Microhabitat use, trophic patterns, and the evolution of brain structure in African Cichlids. Brain Behav Evol 50: 167-182.

Hutcheon JM, Kirsch JW, Garland T (2002): A comparative analysis of brain size in relation to foraging ecology and phylogeny in the chiroptera. Brain Behav Evol 60: 165-180. 
Iwaniuk AN, Nelson JE (2001): A comparative analysis of relative brain size in waterfowl (Anseriformes). Brain Behav Evol 57: 87-97.

Iwaniuk AN, Nelson JE (2002): Can endocranial volume be used as an estimate of brain size in birds. Can J Zool 80: 16-23.

Jelsing J, Rostrup E, Markenroth K, Paulson OB, Gundersen HJG, Hemmingsen R, Pakkenberg B (2005): Assessment of in vivo MR imaging compared to physical sections in vitro: a quantitative study of brain volumes using stereology. Neuroimage 26: 57-65.

Johnson JT, Hansen MS, Wu I, Healy LJ, Johnson CR, Jones GM, Capecchi MR, Keller C (2006): Virtual histology of transgenic mouse embryos for high-throughput phenotyping. PLoS Genet 2:e61.

Kalender WA (2005): Computed Tomography: Fundamentals, System Technology, Image Quality, Applications. Erlangen, Publicis Corporate Publishing.

Kihslinger RL, Lema SC, Nevitt GA (2006): Environmental rearing conditions produce forebrain differences in wild Chinook salmon Oncorhynchus tshawytscha . Comp Biochem Physiol A Mol Integr Physiol 145: 145-151.

Kihslinger RL, Nevitt A (2006): Early rearing environment impacts cerebellar growth in juvenile salmon. J Exp Biol 209: 504-509.

Kim TH, Zollinger L, Shi XF, Rose J, Jeong EK (2009): Diffusion tensor imaging of ex vivo cervical spinal cord specimens: the immediate and long-term effects of fixation on diffusivity. Anat Rec (Hoboken) 292: 234-241.

Kolm N, Gonzalez-Voyer A, Brelin D, Winberg S (2009): Evidence for small scale variation in the vertebrate brain: mating strategy and sex affect brain size and structure in wild brown trout (Salmo trutta). J Evol Biol 22: 2524-2531.

Kotrschal K, Junger H (1988): Patterns of brain morphology in mid-European Cyprinidae (Pisces, Teleostei): a quantitative histological study. J Hirnforsch 29: 341-352.

Kotrschal K, Palzenberger M (1992): Neuroecology of cyprinids: comparative, quantitative histology reveals diverse brain patterns. Environ Biol Fishes 33: 135-152.

Kotrschal, K. Van Staaden MJ, Huber R (1998): Fish brains: evolution and environmental relationships. Rev Fish Biol Fisher 8: 373-408.

Kotrschal A, Sundstrom LF, Brelin D, Devlin RH, Kolm N (2012a): Inside the heads of David and Goliath: environmental effects on brain morphology among wild and growth-enhanced coho salmon Oncorhynchus kisutch. J Fish Biol 81: 987-1002.

Kotrschal A, Rasanen K, Kristjansson BK, Senn M, Kolm N (2012b): Extreme sexual brain size dimorphism in sticklebacks: a consequence of the cognitive challenges of sex and parenting? PLoS One 7:e30055.

LaDage LD, Roth TC 2nd, Pravosudov VV (2009): Biases in measuring the brain: the trouble with the telencephalon. Brain Behav Evol 73: 253-258.

Lecchini D, Lecellier G, Lanyon RG, Holles S, Poucet B, Duran E (2014): Variation in brain organization of coral reef fish larvae according to life history traits. Brain Behav Evol 83: 17-30.

Lisney TJ, Collin SP (2006): Brain morphology in large pelagic fishes: a comparison between sharks and teleosts. J Fish Biol 68: 532-554.

Lisney TJ, Bennett MB, Collin SP (2007): Volumetric analysis of sensory brain areas indicates ontogenetic shifts in the relative importance of sensory systems in elasmobranchs. Raffles Bull Zool 14: 7-15.

Metscher BD (2009a): MicroCT for comparative morphology: simple staining methods allow high-contrast 3D imaging of diverse nonmineralized animal tissues. BMC Physiol 9: 11.

Metscher BD (2009b): MicroCT for developmental biology: a versatile tool for high-contrast 3D imaging at histological resolutions. Dev Dyn 238: 632-640.

Mizutani R, Takeuchi A, Hara T, Uesugi K, Suzuki Y (2007): Computed tomography imaging of the neuronal structure of Drosophila brain. J Synchrotron Radiat 14: 282-287. 
Neues F, Epple M (2008): X-ray microcomputer tomography for the study of biomineralized endo- and exoskeletons of animals. Chem Rev 108: 4734-4741.

Nieuwenhuys R, Meek J (1998): Holosteans and teleosts; in Nieuwenhuys R, Ten Donkelaar HJ, Nicholoson C (eds): The Central Nervous System of Vertebrates. Berlin, Springer, vol 2, pp 759938.

Paterson GLJ, Sykes D, Faulwetter S, Merk R, Ahmed F, Hawkins LE, Dinley J, Ball AD, Arvanitidis C (2014): The pros and cons of using micro-computed tomography in gross and microanatomical assessments of polychaetous annelids. Memoirs Museum Victoria 71: 237-246. from http://museumvictoria.com.au/about/books-and-journals/journals/memoirs-of-museum-victoria/ (accessed February 9, 2015).

Pitnick S, Jones KE, Wilkinson GS (2006): Mating system and brain size in bats. Proc Biol Sci 273: 719724.

Pollen AA, Dobberfuhl AP, Scace J, Igulu MM, Renn SCP, Shumway CA, Hofmann HA (2007): Environmental complexity and social organization sculpt the brain in Lake Tanganyikan cichlid fish. Brain Behav Evol 70: 21-39.

Prajapati SI, Kilcoyne A, Samano AK, Green DP, McCarthy SD, Blackman BA, Brady MM, Zarzabal LA, Tatiparthy AK, Sledz TJ, Duong T, Ohshima-Hosoyama S, Giles FJ, Michalek JE, Rubin BP, Keller C (2011): MicroCT-based virtual histology evaluation of preclinical medulloblastoma. Mol Imaging Biol 13: 493-499.

Purea A, Webb AG (2006): Reversible and irreversible effects of chemical fixation on the NMR properties of single cells. Magn Reson Med 56: 927-931.

Reader SM, Laland KN (2002): Social intelligence, innovation, and enhanced brain size in primates. Proc Natl Acad Sci USA 99: 4436-4441.

Ribi W, Senden TJ, Sakellariou A, Limaye A, Zhang S (2008): Imaging honey bee brain anatomy with micro-X-ray-computed tomography. J Neurosci Methods 171: 93-97.

Rosen GD, Harry JD (1990): Brain volume estimation from serial section measurements: a comparison of methodologies. J Neurosci Methods 35: 115-124.

Safi K, Dechmann DK (2005): Adaptation of brain regions to habitat complexity: a comparative analysis in bats (Chiroptera). Proc Biol Sci 272: 179-186.

Salas C, Broglio C, Rodriguez F, Lopez JC, Portavella M, Torres B (1996): Telencephalic ablation in goldfish impairs performance in a 'spatial constancy' problem but not in a cued one. Behav Brain Res 79: 193-200.

Schambach SJ, Bag S, Schilling L, Groden C, Brockmann MA (2010): Application of micro-CT in small animal imaging. Methods 50: 2-13.

Shultz S, Dunbar RI (2006): Both social and ecological factors predict ungulate brain size. Proc Biol Sci 273: 207-215.

Stowell RE (1941): Effect on tissue volume of various methods of fixation, dehydration, and embedding. Biotech Histochem 16: 67-83.

Striedter GF (2005): Principles of Brain Evolution. Sunderland, Sinauer Associates. Ullmann JFP, Cowin G, Collin SP (2010): Quantitive assessment of brain volumes in fish: comparison of methodologies. Brain Behav Evol 76: 261-270.

Uylings HBM, van Eden CG, Hofman MA (1986): Morphometry of size/volume variables and comparison of their bivariate relations in the nervous system under different conditions. J Neurosci Methods 18: 19-37.

Van Staaden MJ, Huber R, Kaufman LS, Liem KF (1995): Brain evolution in cichlids of the African Great Lakes: brain and body size, general patterns, and evolutionary trends. Zoology 98: 165-178.

Vargas JP, Rodriguez F, Lopez JC, Arias JL, Salas C (2000): Spatial learning-induced increase in the argyrophilic nucleolar organizer region of dorsolateral telencephalic neurons in goldfish. Brain Res 865: 77-84. 
Wagner HJ (2001a): Sensory brain areas in mesopelagic fishes. Brain Behav Evol 57: 117-133.

Wagner HJ (2001b): Brain areas in abyssal demersal fishes. Brain Behav Evol 27: 301-316.

Weil A (1928): The measurement of cerebral and cerebellar surfaces. V. The determination of the shrinkage of the surface of different vertebrate brains. Arch Neurol Psychiatry 20: 834-835.

White GE (2015): Microhabitat use affects brain size and structure in intertidal gobies. Brain Behav Evol, Epub ahead of print.

Wilson ADM, McLaughlin RL (2010): Foraging behaviour and brain morphology in recently emerged brook charr, Salvelinus fontinalis. Behav Ecol Sociobiol 64: 1905-1914.

Wiper ML, Britton S, Higgs DM (2014): Early experience and reproductive morph both affect brain morphology in adult male Chinook salmon (Oncorhynchus tshawytscha) . Can J Fish Aquat Sci 71: 1430-1436.

Wullimann MF, Rupp B, Reichert H (1996): Neuroanatomy of the Zebrafish Brain: A Topological Atlas. Basel, Birkhauser.

Wullimann MF, Mueller T (2004): Teleostean and mammalian forebrain contrasted: evidence from genes to behavior. J Comp Neurol 475: 143-162.

Young LJ, Wang Z, Insel TR (1998): Neuroendocrine bases of monogamy. Trends Neurosci 21: 71-75. 